(Seine Praxis nöthigte ihn jedoch von der Fortsetzung seiner Arbeiten abzusehen.)

Das war der Beweggrund für mein Ersuchen, dem er sogleich wohlwollend entgegen kam; es ist ihm in der That gelungen, der Aufgabe mit Huilfe selbstständig construirter Apparate gerecht zu werden.

Die Beobachtungen schienen mir von so hohem Interesse zu sein, dass ich sehr gern der Aufforderung des Herrn Dr. C. Sandborg, mit ihm an den Untersuchungen theilzunehmen, Folge leistete. Diese haben bisher dazu gedient, Hauptfragen betreffs des Mechanismus des Herzens zu erörtern.

(Aus dem physiologischen Institut zu Christiania.)

\title{
Studien über den Mechanismus des Herzens.
}

\section{Von}

Dr. C. Sandborg und Prof. Worm Müller.

Hierzu Tafel III, IV u. V.

\section{Die Apparate und die Anordnung der Versuche.}

Aus naheliegenden Gründen würde es von grösstem Interesse sein, Menschenherzen für die Untersuchungen des Klappenspiels anzuwenden.

Da aber diese in der Regel nicht in genügend frischem Zustande zu haben sind, und da es iuberdies wïnschenswerth erschien, die Beobachtungen an Herzen von möglichst grossen Dimensionen anzustellen, wurde als geeignetes Untersuchungsobject das Ochsenherz gewählt.

Man ist im Voraus zu der Annahme berechtigt, dass die Resultate nicht wesentlich verschieden ansfallen können, mag man Ochsen- oder Menschenherzen anwenden, aber um grössere Sicher- 
heit hierüber zu erhalten, wurden vergleichende anatomische Untersuchungen an den beiden Herzen angestellt. Diese ergaben eine bedeutende Uebereinstimmung in dem Bau der beiden Herzen (sowohl der Klappen als auch der Muskulatur). Der Unterschied war wesentlich nur ein quantitativer ${ }^{1}$ ).

- Um das Ochsenherz zu den vorliegenden Untersuchungen benutzen zu können, erscheint es vor allem nothwendig, zu warten, bis die Todtenstarre im Wesentlichen gehoben ist ${ }^{2}$; ; in diesem Zustande ist nämlich das Herz so stark zusammengezogen, dass sich rhythmische Bewegungen desselben nicht bewerkstelligen lassen. Um das Lösen der Starre zu beschleunigen, wurde das Herz durch Manipulationen erweicht, welche am ehesten mit der Massage zn. vergleichen sind. Um Fäulniss zn verhindern, sowie um die Integrität der Klappen zu conserviren, wurde das Herz nun in einer Auflösung von Borsäure $(1 / 2 \%)$ aufbewahrf, welche es nach 1 - bis 2tägiger Einwirkung zu Versuchen besonders geeignet machte. Die Muskulatur ist dann schlaff, aber, wie es scheint, unbeschädigt; die Klappen sind sufficient und bleiben es in den folgenden 4 bis 5 Tagen.

Die nächste Aufgabe, welche sich jetzt darbot, war die, mit Hiilfe des so präparirten Herzens eine vollständige Leitung zu Stande zu bringen; also eine Verbindung zwischen der Aorta und den Venae cavae auf der einen Seite und zwischen der Arteria pulmonalis und den Venae pulmonales anf der anderen Seite herzustellen, sowie gleichzeitig die Leitung an den Stellen durchsichtig zu machen, an denen der Strom die Klappen passirt. $\mathrm{Zu}$ diesem Zwecke wurde die eine Vena cava (gewöhnlich superior) unterbunden, während durch die andere ein $9,5 \mathrm{~cm}$ langes, inwendig vernickeltes Messingrohr hineingeführt warde (siehe Taf. III, Fig. 1), dessen Lumen einen Durchmesser von 4,8 cm hatte. Dieses Rohr wurde so tief in das rechte Atrium eingeführt, dass sich die untere Oeffnung (a) circa $0,7 \mathrm{~cm}$ über dem Ostium atrioventriculare befand. In dem Theil des Rohres, welcher im Atrium lag, waren ring:s herum ungefähr 20 Oeffunngen $(\mathrm{bb})$ vón $0,5 \sim 0,7 \mathrm{~cm}$ Durch-

1) In dem Menschenherzen haben die Wände der Herzkammerhöhlen ein mehr trabeculäres Aussehen, im Ochsenherzen sind dieselben mehr glatt.

2) Man kann das Herz nicht gleich nach dem Tode verwenden, da die Todtenstarre rasch eintritt. 
messer angebracht, damit sowohl das Füllen wie das Entleeren der Aurikeln mit Leichtigkeit stattfinden konnte. Die obere Oeffnung (c) war mit einer ebenen Glasplatte verschlossen, durch welche man die Tricuspidalklappe bis zu ihrer Insertion an den Papillarmuskeln übersehen konnte.

Da das Rohr einen Theil der Leitung ausmachen musste, war es mit einer Seitenöffnung (d) in dem Theile versehen, der ausserhalb des Atriums sich befand. In dieser Oeffnung war ein Rohr (e) mit einem Durchmesser von ungefähr $3 \mathrm{~cm}$ angebracht; mit Hülfe eines Kautschuckrohres von ungefähr gleichem Durchmesser stand dieses mit einem grösseren Behälter A (siehe Taf. III, Fig. 1) in Verbindung. Ein Rohr von derselben Construction wurde durch die Venae pulmonales in das linke Atrium eingeführt; das Seitenrohr communicirte hier durch einen Kautschuckschlauch mit dem zweiten Behälter $\mathrm{B}^{1}$ ) (siehe Taf. III, Fig. 3).

Durch die Arteria pulmonalis und die Aorta wurden ebenfalls röhrenförmige Specula (siehe Taf. III, Fig. 2) angebracht, welche sich von den vorhergehenden nur dadurch unterschieden, dass sie nicht in ihrem unteren Theile durchlöchert waren, sowie dadurch, dass die Oeffnungen einen etwas kleineren Durchmesser, nämlich $4,2 \mathrm{~cm}$, hatten. Die unteren Oeffnungen dieser Rohre befanden sich unmittelbar über den oberen Befestigungspunkten der balbmondförmigen Klappen. (Die halbmondförmigen Klappen und die nächst gelegenen Theile der Arterien und der Herzkammern liessen sich nun vollständig iubersehen.) Die Seitenrohre standen auch hier mittelst Kautschuckschläuche mit den beiden Behältern in Verbindung, so dass der Kautschuckschlauch von dem Ansatzrohr in der Aorta zu dem Behälter A, und der Kautschucksehlauch von dem Ansatzrohr in der Arteria pulmonalis zu dem Behälter $B$ fübrte. Dadurch war eine continuirliche, dem grossen and kleinen Kreislauf entsprechende Leitung hergestellt. Die ganze Leitung und das Herz wurden nun mit Wasser gefüllt. Bei diesem Füllen ist es nothwendig, die eingeschlossene Luft vollständig herauszutreiben. $\mathrm{Zu}$ diesem $\mathrm{Zwecke}$ waren an den röhrenförmigen Speculae in der Nähe der oberen Oeffnungen ganz kurze, mit leicht an- und

1) Beide Behälter waren von gleicher Grösse und jeder derselben konnte ungefähr 4 Liter fassen. 
abschraubbaren Kapseln versehene Ansatzrohre (f) angebracht. Während des Füllens wurden die Kapseln abgeschraubt; die Luft entwich durch die Oeffnungen, worauf diese geschlossen wurden.

Es eruibrigte nun noch gleichzeitig ein rhythmisches Zusammenziehen der beiden Herzhälften zu erzeugen, um dadurch einen continuirlichen Strom und Klappenspiel hervorzubringen.

Der Apparat, welcher diesen Zweck erfüllen sollte, musste dabei auch beide Herzkammern so comprimiren, dass die künstliche Raumverringerung der natürlichen entsprach. Als Massstab zur Beurtheilung wurden die Erfahrungen tiber das Verhalten der Herzkammern während der Todtenstarre benutzt. Es werden dieselben in dem letzten Abschnitt dieser Abhandlung mitgetheilt werden. Es ist uibrigens nothwendig, schon hier hervorzuheben, dass die Experimente dargethan haben, dass weder die linke noch die rechte Herzkammer selbst durch die stärkste Contraction ihren Inhalt vollständig entleeren können.

Es gelang in der That, einen Apparat zu construiren, mit dessen Hitlfe man in Intervallen gleichzeitig beide Herzkammern - so zusammendrücken konnte, dass die Verkleinerung der Cavitäten und ihre Formveränderung den Verhältnissen bei der Todtenstarre einigermassen adaequat waren. Der Apparat ist so beschaffen, dass man je nach Bedarf die Intervalle verkürzen oder verlängern kann; derselbe ermöglicht auch eine künstliche Dilatation, welche bei den bisher ausgefiuhrten Versuchen nicht in Anwendung gebracht worden ist. Wie der Apparat wirkt, geht ohne Weiteres aus dem Vorhergehenden hervor, aber, wie der Apparat construirt ist, um auf diese Weise zu wirken, kann nur mit Hülfe einer detaillirten, von Zeichnungen begleiteten Beschreibung klargemacht werden. Dieses soll einer späteren Arbeit vorbehalten bleiben, welche die Herztöne und die Circulation durch die Lungen umfassen wird.

Der Leser wird eine Uebersicht über die Anordnungen der Versuche erhalten, wenn er Taf. III, Fig. 3 betrachtet, welche nach der Photographie des sich in Thätigkeit befindenden Apparates angefertigt wurde; a ist die obere Oeffnung des Rohres der Arteria pulmonalis, $b$ die des linken Atriums, $c$ die der Aorta und d die des rechten Atriums; $a^{\prime}, b^{\prime}, c^{\prime}, d^{\prime}$ bezeichnen die entsprechenden Seitenrohre mit ihren Kautschuckschläuchen; $a^{\prime} b^{\star}$ stehen mit dem 
Behälter $B$ und $e^{\prime} \mathbf{d}^{\star}$ mit dem Behälter $A$ in Verbindung. Der Druck ist etwas grösser in dem Bebälter B als in dem Behälter A; ferner sieht man, dass sich die Mündung der Schlauchleitung von der Aorta in dem Behälter A etwas höher als das Wasserniveau des Behälters B befindet. Bei dieser Versuchsanordnung sind die halbmondförmigen Klappen geschlossen und die Atrioventricularklappen offen; diese Stellung müssen die Klappen beim Anfange des Experimentes einnehmen.

Lässt man nun den Apparat in Intervallen wirken, z. B. 50 bis $60 \mathrm{Mal}$ in der Minute, so kann man direct einen vollständigen Kreislanf mit Klappenspiel und Pulsation beobachten. Die Systole der Herzkammern wird durch den Apparat erzengt, ihre Diastole und die der Atrien durch den continuirlichen Strom, die Systole der Atrien riuhrt von der Druckverringerung: in den Herzkammern während der Diastole her. Da die Klappen in ihrer ganzen Ausdehnung übersehen werden können, und sie längere Zeit hindurch mit Präcision fungiren, kann man leicht methodische Observationen vornehmen; die wichtigsten derselben sollen zunächst in Folgendem mitgetheilt werden.

II.

Das Klappenspiel und das Verhalten der Atrien und Ventrikeln.

A. Das Klappenspiel.

1. Die Tricuspidal- und die Mitralklappe schliessen sich auf die Weise, dass die centralen Theile gegen das Niveau der Ostia hinaufgehoben werden and sich mit um- und nach unten gebogenen Rändern zusammenlegen, ef. Taf. III, Fig. 4. (ch. t. sind die Chordae tendineae; aa. ist der Frontalschnitt der Aorta, auf deren hinterer Wand man zwei durchschnittene Klappen bb sieht).

Da es wegen der Umbiegung der centralen Partien Flächen sind, welche sich zusammenlegen, wird der Verschluss in einer grösseren Ausdehnung hergestellt. Ferner wird der Verschluss auch dadurch gesichert, dass die umgebogenen Randpartien zahnförmig in einander eingreifen, so dass diese Klappen, welche an 
und fur sich sehr stark gebaut sind und ausserdem durch Chordae tendineae gehindert werden, in die Vorkammern umzuschlagen, einen starken Druck aushalten können.

Die Chordae tendineae (ch. t.), welche von den umgebogenen Rändern (also ungefähr von der Mittellinie der Ostia) nach únten in die Herzsubstanz gehen, stehen wäbrend des Schliessens frei in den Höhlen, siehe die eben genannte Figur, welche schematisch (in verkleinertem Massstabe) einen Frontalschnitt eines Ochsenherzens durch die Mitte der linken Herzkammer und der Aorta darstellt (die Atrioventricularklappe künstlich verschlossen, der Ventrikel im Rigor mortis und die Aorta systolisch erweitert).

Sowohl das Schliessen der Klappen wie die Art und Weise, auf welche sie sich zusammenlegen, finden in Folgendem ihre Erklärung.

In demselben Augenblicke, in dem die Systole der Herzkammern anfängt, entsteht eine gegen die Basis des Herzens gehende Strömung des Blutes in den Ventrikeln. Von diesem Strome wird derjenige Theil, welcher sich zwischen den inwendigen (resp. oberen) Flächen der Atrioventricularklappen befindet, nur einen unbedeutenden Druck auf diese Flächen hervorbringen, weil der Widerstand für den Ablauf in die Atrien gering ist; dagegen wird das übrige Blut (welches rings herum die Klappen umgibt), theils, weil es auf grossen Widerstand in den Arterien stösst und theils, weil es durch die winkelförmigen, nach oben verschlossenen Räume, welche die Atrioventricularklappen und die Ventrikelwände zusammen bilden, in seinem Ablauf gehindert wird, einen bedeutenden Druck auf.die äusseren resp. unteren Flächen der Atrioventricularklappen ausiiben. Hieraus resultirt, dass die Zipfel der Klappen gegen einander getrieben werden und die Ostia verschliessen. Da der Unterschied der Drücke in demselben Augenblicke eintritt, in dem das Blut seine Bewegung gegen die Basis beginnt, so wird das Schliessen auch innerhalb eines ganz kurzen Zeitraumes $\mathrm{nach}$ dem Beginn der Systole vor sich gehen; das Quantum Blut, welehes in die Atrien zurückströmt, kann demnach nur unbedenitend sein.

Nach dieser Erklärung ist das Sehliessen einzig und allein durch das in den Herzkammern enthaltene Blut (Wasser) bedingt, welches, so lange das Herz zusammengezogen, gegen die unteren Flächen der Klappen hinaufgedrückt wird. 
Wenn man nun bedenkt, 1) dass die äusseren Ränder der Klappen unbeweglich an die Ostia befestigt, während sich die inneren Ränder innerhalb derjenigen Grenzen, welche die Chordae tendineae zulassen, bewegen können, und 2) dass die Flächen so gross sind, dass sie in einer gewissen Ausdehnung einander zu decken vermögen, sowie 3) dass die Chordae tendineae weniger nachgeben, als die Klappen selbst, so hat man die nöthigen Elemente, um vollständig zu verstehen, wie ein starker Druck des Blutes gegen die Aussen- (resp. unteren) Flächen der Klappen ein Schliessen derselben mit um- und nach unten gebogenen Rändern hervorzubringen im Stande ist. Dass diese zahnförmig in einander greifen, rührt davon her, dass die dünneren und biegsameren Partien zwischen den Insertionsspitzen der Chordae tendineae in Falten gelegt, während die Spitzen selbst in Folge ihrer grösseren Dicke and Steifheit von dem Blute hervorgetrieben werden, und Vorsprünge oder Zähne bilden. Das Falten findet in Folgendem seine Erklärung: Die centralen Partien der Klappen gehen von der Ringform, welche sie bei der offenen Klappenstellung einnehmen, bei dem Schliessen der Klappen in eine mehr lineare Form über, und müssen sich demnach, um in dem kleineren, der Mittellinie der Ostia entsprechenden Raume Platz zu finden, in Falten legen.

2. Die halbmondförmigen Klappen verschliessen die respectiven Ostia, indem sie sich sowohl mit ihren Rändern wie mit einem Theile (ca. 1/6) ihrer unteren (doppelt convexen) Flächen zusammenlegen. Hier sind es also die unteren Flächen, welche einander beriihren; bei den Atrioventricularklappen dagegen sind es die oberen.

In dem Augenblicke, in welchem die Herzkanmern ihre Erweiterung beginnen, wird der Widerstand sowohl des unter den halbmondförmigen Klappen wie des unter den Atrioventricularklappen stehenden Blutes ") gehoben, und die Drücke in den Atrien und Arterien gewinnen die Oberhand. Daraus folgt, dass sich das in denselben enthaltene Blut in der Richtung nach unten gegen die Spitze des Herzens in Bewegung setzen wird.

Diese Bewegung nach unten wird, was die Atrien betrifft, mit der Füllung der Herzkammern, was die Arterien anbelangt, mit dem Schliessen der halbmondförmigen Klappen

1) Vergl. S, 425. 
endigen. Di eses letztere lässt sich auf folgende Weise erklären. Der centrale Theil des Blutes, welches sich in den Arterien in gleicher Höhe mit den halbmondförmigen Klappen befindet, also der Theil, welcher in dem Raume zwischen den offenen nach aufwärts gesehlagenen Klappen liegt, wird ungehindert seinen Ablauf in die Herzlsammern finden, während die mehr peripherischen Theile desselben gegen die äusseren Flächen der (ziemlich hohen) sackförmigen Semilunarklappen anstossen und in ihrem weiteren Laufe gehindert werden. Hierdurch entsteht eine Differenz in den Drïcken auf beide Flächen der Klappen, indem man den Druck auf die inwendigen resp. unteren Flächen fast mit Null bezeichnen kann, während der Druck auf die auswendigen (resp. oberen) Flächen immer grösser sein wird. Aber die Folge dieser ungleichen Drücke ist natürlicherweise die, dass die freien Ränder der Klappen gegen einander geführt werden, und die Ostia verschliessen.

Aus der hier gegebenen Erklärung des Schliessens der halbmondförmigen Klappen geht hervor, dass dasselbe unter einer zurïckgehenden Bewegung des in der untersten Partie der Arterien befindlichen Blutes vor sich geht, und dass es erst stattfindet, nachdem die Diastole der Herzkammern ihren Anfang genommen hat. Aus dem bei den Atrioventricularklappen angeführten Grunde wird anch hier, während das Schliessen vor sich geht, nur ein unbedeutendes Quantum Blnt zurückfliessen.

Der Bau der halbmundförmigen Klappen deutet darauf hin, dass sie einen weit geriugeren Druck als die Atrioventricularklappen anszuhalten haben. Die Valvulae semilunares sind nämlich sehr dïnnwandig and ausserdem nicht mit Chordae tendineae versehen.

Um eine Vorstellung von der Grösse der Drücke zu bekommen, welchen die Klappen im Augenblick des Schliessens, also in dem für die Probe auf die Stärke der Klappen kritischesten Zeitpunkt ausgesetzt sind, machen zu können, ist es nothwendig, zu erinnern, dass das Strecken der Arterien in longitudineller wie in transverseller Richtung am Ende der Systole nur einen Theil derjenigen Kraft repräsentirt, mit welcher sich die Herzkammern zusammenziehen, und dass nur dieser Theil eine Bedeutung für den Druck auf die Klappen in geschlossenem Zustande haben kann. Erinnert man sich ferner daran, dass wiederum dieser Theil, in 
dem Augenblicke, in dem sich die ausgedehnten Arterienwände zusammenzuziehen anfangen, in zwei Richtungen, nämlich in einer peripherischen 1 ) und einer centralen Richtung wirkt, also sich gewissermassen spaltet, und dass es nur dieser letatere in centraler Richtung wirkende Theil ist, welcher den Druck gegen die Klappen ausuibt, so wird man auch leicht einsehen, dass dieser Druck nach den wiederholten Auswechslungen, oder richtiger gesagt, Ausgleichungen der Hauptkraft bedeutend geringer als diese sein muss. Die halbmondförmigen Klappen brauchen deshalb durchaus nicht so stark gebaut zu sein wie die Atrioventricularklappen und bedürfen (wegen des geringen Druckes und ibrer Sackform) keiner Chordae tendineae, um an dem Umschlagen gehindert zu werden.

Um die Drücke, welche auf die Klappen im Augenblicke des Schliessens ausgeübt werden, schematisch zu veranschaulichen, dient Taf. III, Fig. 5, welche einen Frontalschnitt durch die Mitte der linken Herzkammer und der Aorta (in verkleinertem Massstabe, der Fig. 4 entsprechend) in dem Momente darstellt, in dem sich die halbmondförmigen Klappen schliessen).

(aa sind die durchschnittenen, stark zusammengezogenen Arterienwände (vgl. Fig. 4), bb zwei durchsehnittene Klappen.)

Die in dem Vorhergehenden gegebene Darstellung des Klappenschlusses weicht erheblich von derjenigen Ceradini's ${ }^{2}$ ) ab, welcher meint, dass sich die Klappen schliessen, noeh bevor die Diastole der Herzkammern ihren Anfang nähme, und dass in Folge dessen jedes Zurïckströmen des Blates in die Herzkammern verhindert werde.

Den theoretischen Beweis für seine Behauptung holt Ceradini theils von den Erfahrungen über die Saug- und Druckpumpen, theils von den Gesetzen für die Bewegung von Flüssigkeiten in starren Röhren, und die Berechtigung, diese Gesetze auf die Strömungsverhältnisse in dem Organismus zu übertragen, stïtzt er auf Experimente, angestellt mit einer ausgeschnittenen Art. pulmonalis, welche durch eine Leitung von Glasröhren mit einem Saug-

1) Der Druck in peripherischer Richtung dient, wie bekannt, dazu, den continuirlichen Strom zu unterhalten.

2) Dr. J. Ceradini: „Der Mechanismus der halbmondförmigen Herzklappen. Leipzig 1872". S. 53-64. 
und Druckapparate in Verbindung stand (1. c. S. 38-41, S. 56-59 sowie Fig. 1 der beigefügten Tafel).

Abgesehen davon, dass die hydraulischen Gesetze, auf welche sich Ceradini beruft, nicht unangreifbar sind, ist die Anordnung der Versuche so abweichend von den Verhältnissen in dem Organismus, dass sich die aus den Observationen gezogenen Schlïsse nicht ohne Weiteres auf diesen übertragen lassen; dies hat jedoch Ceradini gethan.

Da sich die Darstellung Ceradini's unter den Physiologen und Pathologen eine nicht unbedeutende Zahl Anhänger erworben hat, dürfte es von Interesse sein, dieselbe näher zu beleuchten.

In dem Zeitraume zwisehen dem Ende der Systole und dem Anfang der Diastole soll - analog mit dem, was nach Ce radini unbedingt bei Flïssigkeiten, welche in star ren Röhren mit Hülfe eines Stempels in Bewegung gesetzt werden, stattfindet - eine Spaltung des (in der Aorta und in der Art. pulmonalis) sich vorwärts bewegenden Stromes in einen axial vorwärtsschreitenden und in einen peripherisch zurücklanfenden geschehen; der Letztere ist es nun, weleher nach Ceradin i das Schliessen der Klappen noch vor Beginn der Diastole (S. 53-54) bewirkt; das Verhalten des axialen Stromes zu diesem Schliessen bespricht er dagegen gar nicht. Es liegt deshalb nahe, anzunehmen, dass Ceradini von der Ansicht ausgegangen ist, dass das Blut am Ende der Systole iiber die oberen freien Ränder der halbmondförmigen Herzklappen hinausgekommen, so dass der hier auftretende axiale Strom uiber den Bereich hinausgebracht sei, in welchem er auf die Klappen eine Wirkung ausüben könne, während der gleichzeitig auftretende peripherische Strom so weit zurïck sein sollte, dass er den Wänden und dem Boden des Sinus Valsalvae entlang die concaven Flächen der Klappen erreichen und diese schliessen könnte. Dass sich Ceradini wirklich so etwas gedacht hat, geht aus seinen Ausspriichen S. 54 hervor; dort sagt er nämlich, dass das Schliessen der Klappen auf analoge Weise selbst dann geschieht, wenn vielleicht am Ende der Systole etwas Blut unter den halbmondförmigen Herzklappen in der Kammer zurüekbleiben sollte. Diese Möglichkeit würde Ceradini kaum hervorgehoben haben, wenn er nicht, wie bemerkt, zunächst von der Voraussetzung ausgegangen wäre, dass das Blut am Ende der Systole bei den Klappen voriiber gekommen wäre. 
Aber ausser, dass diese Voraussetzung rein hypothetisch und ganz unwahrscheinlich, ist auch die unter dieser Voraussetzung gegebene Erklärung des Schliessens der Klappen mangelhaft. So ist z. B. keine Rücksicht auf diejenigen Theile des zurücklaufenden peripherischen Stromes genommen worden, welche, während gleichzeitig andere Theile desselben in die Sinus Valsalvae hineinströmen, die triangulären Räume durchlanfen miissen, die sich bei den vollständig geöffneten Klappen zwischen deren freien Rändern vorfinden; hierdurch wird selbstverständlich dem Schliessen der Klappen entgegengewirkt. Ausserdem ist die Grösse des Druckes, welche zum Verschluss der Klappen erforderlich ist, nicht einmal annähernd angegeben; nach einiger Erwägung wird man nämlich einsehen, dass der Druck des zurücklaufenden Stromes unter der hier gemachten Voraussetzung nicht beträchtlich sein kann. - Dass unter diesen Umständen keine Regurgitation Statt finden sollte, ist kanm glaublich, weil derjenige Theil des peripherischen Stromes, welcher, wie oben bemerkt, zwischen den Klappen hinunterläuft, nur in einem darunterliegenden Raume Platz finden kann.

Wenn aber Ceradini annimmt, dass derselbe Schliessungsmechanismus auch in dem Falle stattfindet, wenn am Ende der Systole etwas Blut unter den Semilunarklappen zurückbleiben sollte, so scheint diese Annahme so unhaltbar, dass sie kaum auf irgend eine Weise vertheidigt werden kann. Nach Ceradini soll nun auch in diesem, unter den Klappen befindlichen Blute am Ende der Systole eine Spaltung in einen axial vorwärtsgehenden und einen peripherisch rückwärtsgehenden Strom stattfinden (1. c. S. 54); er gibt zu, dass der axiale Strom dem Schliessen entgegenwirken wird, legt aber das Hauptgewicht auf die auf der Ober- and Unterseite zurücklaufenden peripherischen Ströme, welche die Klappen nach unten zum Verschliessen treiben sollen. Nun sagt aber Ceradini (S. 52), dass bei der Bewegung: von Flüssigkeiten in starren Röhren ein rascherer centraler und ein langsamerer peripherischer Strom entsteht, und dass diese Ströme in Folge des Trägheitsmomentes noch eine Zeit lang anhalten, nachdem die bewegende Kraft zu wirken aufgehört habe. Da nun Ceradini ferner (1. c. S. 53) sagt, dass sich der Strom in dem Augenblicke, in dem die bewegende Kraft aufhört, in einen axial vorwärtsgehenden und einen peripherisch rückwärtslaufenden spal- 
tet, und da es einleuchtend, dass der axiale Strom nichts anderes als der oben besprochene centrale, und dass der ritckwärtslaufende (im Wesentlichen) nichts anderes, als der peripherische ist, - welcher, um den durch das Vorlaufen des rascheren, centralen Stromes entstandenen luftleeren Raum auszufüllen, in einen zurücklaufenden verwandelt worden ist - so muss auch zugegeben werden, dass es die Richtung des axialen Stromes ist, welche hier die Stellung der Klappen bestimmt. Da dieser Strom in der Richtung nach aufwärts geht, müssen die unteren Flächen der semilunaren Klappen nothwendigerweise auseinander gehalten werden, und die Klappen somit offen bleiben. Wenn sich demnach am Ende der Systole unter den halbmondförmigen Klappen Blut vorfindet, und wenn sich in diesem Blute ein axialer und ein peripherischer Strom bildet, so werden die Klappen ge rade aus diesen Gründen, jedenfalls bis die Diastole ihren Anfang nimmt, geöffnet bleiben.

$$
\text { 3. "e) Das Oeffnen der semilunaren Klappen. }
$$

Gleich bei Beginn der Systole fangen die Klappen sich zu öffnen an; man beobachtet dann zunächst, dass ihre Flächen von einer mehr gebogenen zu einer flacheren Form iubergehen. Indem nun die Klappen heraufgeschlagen werden, verlieren die Flächen ihr ebenes Aussehen und werden leise in longitudinaler Richtung zusammengefaltet. Bei keinem Versuche ist es gelungen, selbst durch forcirten Druck, die oberen (biconcaven) Flächen der Klappen mit den Wänden der Sinus Valsalvae ${ }^{1}$ ) in Berührung zu bringen; ès ist auch nicht gelungen, die oberen Ränder der Klappen dahin zu bringen, sich ganz an die Arterienwände zu legen. Es hat sich allerdings ereignet, dass ein kleinerer Theil des Randes einer einzelnen Klappe gegen die Wand anschlug; aber die Berïhrung war höchst unvollständig.

Die Observationen beweisen fermer, dass das Oeffnen der Klappen successive und leise geschieht; es erklärt sich dies dadurch, dass die geschlossenen Klappen nicht senkrecht auf der Längenaxe der Arterien stehen; die unteren, biconvexen Flächen,

1) Es ist ohne Weiteres klar, dass die oberen Flächen der Semilunarklappen biconvex werden müssten, wenn sie sich an die Sinus Valsalvae anlegen sollten; ein solcher Contact wäre daher a priori unwahrscheinlich. 
welche hier in Betracht kommen, haben nämlich eine schiefe Richtung, so dass sich die Blutcolonne, welche am Ende der Diastole unter den Semilunarklappen steht, am Anfange der Systole zwischen die freien Ränder derselben in Form eines dreieckigen Keiles mit hohlen Seiten (a, b, c, Taf. V, Fig. 1), den unteren biconvexen Flächen der Semilunarklappen entsprechend, hineindrängt.

Während die Klappen vollständig geöffnet sind, kann der Druck sowohl auf ibre äusseren (resp. oberen), wie ihre inneren (resp. unteren) Flächen an und für sich bedeutend sein, aber da diese beiden Flächen nur durch eine papierdünne Zwischenwand getrennt, sowie von Blut umgeben sind, das auf die correspondirenden (zwei einander gegenüberliegenden) Punkte dieser Flächen wohl im Wesentlichen denselben Druck ausübt, so werden die Semilunarklappen in dieser Stellung eigentlich nur eine Compression ihrer Substanz auszuhalten haben und brauchen deswegen hier nur eine dem entsprechende Resistenz zu besitzen.

B) Das Verhalten der Aortaklappen den Art. coronariae cordis gegenüber.

Bei den Versuchen zeigte es sich, dass die Pulse in den Art. coronariae cordis mit dem Puls in der Aorta synchronisch sind. Wiewohl es demnach einlenchtend sein sollte, dass dieselben nicht während der Diastole gefüllt werden, so dürfte es doch von Interesse sein, in Kürze das Verhalten der Aortaklappen den Art. coronariae cordis gegenüber zu besprechen.

Was das Ochsenherz anbelangt, so würde die Art. coronaria dextra (posterior) von der entsprechenden Klappe bedeckt werden können, wenn sich der Rand derselben vollständig bis an die Wand zurückschlagen würde; dagegen kann dies nach den $\mathrm{Ob}$ servationen mit der Art. coronaria sinistra (anterior) nicht der Fall sein ${ }^{1}$ ). Dieselbe entspringt in der Nähe der rechten Eeke des linken (vorderen) Sinus Valsalvae und liegt in der Regel mit wenigstens $1 / 3$ ihres Lumens über den obersten Befestigungspunkten der entsprechenden Klappe. Die Arteria coronaria dextra (posterior) dagegen entspringt ungefähr aus der Mitte des rechten

1) Die anatomischen Verhältnisse des Menschenherzens haben sich gewöhnlich im Wesentlichsten als damit übereinstimmend gezeigt. 
Sinus Valsalvae and liegt niedriger als die entsprechenden oberen Befestigungspunkte der dazı gehörigen Klappe. Man könnte demnach vielleicht glauben, dass die Art. coronaria posterior während der Systole von der Klappe vollständig versehlossen und deshalb wahrscheinlich während der Diastole gefüllt würde. Ein derartiger Verschluss findet jedoch nicht Statt; abgesehen davon, dass die Pulse in beiden Art. coronariae synchronisch mit dem Puls der Aorta sind, haben ja die Observationen bereits gezeigt, dass die Klappen nie vollständig gegen die Wände zurückgeschlagen werden können ${ }^{1}$ ).

\section{B. Das Verhalten der Atrien und Ventrikeln.}

\section{Das Verhalten der Atrien.}

Wenn die Vorkammern im Stande sein sollen mit Leichtigkeit alles Blut aufzunehmen, welches während der Systole ventriculorum hineinströmt, so müssen ihre Wände einen hohen Grad von Nachgiebigkeit besitzen. Die Versuche ergaben mit vollständiger Sicherheit, dass die Wände im Ganzen genommen im Besitze einer derartigen Dehnbarkeit sind; man sah mit der grössten Klarheit, dass besonders die Prolongationen, welche Aurikeln genannt werden, während der Systole ventriculorum immer mehr und mehr anschwollen, so dass die Atrienräume bedeutend zunahmen. Es kann z. B. angeführt werden, dass jedes Atrium eines grösseren Ochsenherzens $90-100 \mathrm{ccm}$ Wasser ohne zu bersten oder seine Elasticität zu verlieren, mit Leichtigkeit aufnehmen konnte, während gleichzeitig ein entsprechendes Quantum aus jedem Ventrikel herausgetrieben wurde. Der Zufluss darf

1) Die Semilunarklappen zeigen ein etwas abweichendes Verhalten bezüglich ihrer Insertion. Die rechte Aortenklappe heftet sich nicht blos an die eigentliche Arterienwand, sondern auch mit ihrer unteren Fläche an ein in das Lumen der Aorta nicht unbedeutend vorspringendes muskulöses Promontorium; von den beiden anderen Klappen zeigt nur die hintere ein einigermassen älnliches Verhalten in ihrer rechten Hälfte (doch besteht hier das Promontorium aus Fibrocartilago), während die zweite Hälfte und die vordere (linke) Klappe überall direct von der Wand ausgehen. Sämmtliche Klappen in der Arteria pulmonalis zeigen dagegen dasselbe Verhalten wie die rechte Klappe in der Aorta; es sind aber hier die muskulösen Promontorien weniger hervortretend; die physiologische Bedeutung derselben ist noch nicht in's Reine gebracht. 
indess nur unter einem verhältnissmässig geringen Drucke vor sich gehen, da die Aurikeln an einzelnen Punkten leicht barsten, wenn die Druckhöhe in den Behältern gesteigert wurde.

Mit dem Beginn der Diastole nahmen die Cavitätenräume wieder ab, weil jetzt das Blut in die Herzkammern hineinströmte.

\section{Das Verhalten der Ventrikeln.}

c) Die Raumverkleinerung.

In Folge der Contraction der Muskelsubstanz werden die Cavitäten verkleinert, und das Blut wird aus den Herzkammern herausgetrieben. Mehrere Physiologen haben darzuthun versucht, dass die Blutmenge, welehe durch die Systole ventriculorum entleert wird, demjenigen Quantum entspricht, welches die Herzkammern fassen können, mit anderen Worten, dass die Ventrikeln während der Systole ihren Inhalt vollständig entleeren sollten. Aber wenn man bedenkt, dass sich die Calcuile ïber die Blutmengen, welche bei jeder Systole hinausgetrieben werden, auf Experimente mit lebenden Thieren stiutzen, wobei viele Complicationen auftreten, während die Berechnungen der Blutmenge, welche die Ventrikeln fassen, auf Messungen der Cavitäten im schlaffen Zustande nach dem Tode beruhen, so wird es klar erscheinen, dass die Fehlerquellen so gross sind, dass man keinen bestimmten Schluss ziehen kann.

Die Frage, inwieweit die Ventrikeln während der Systole vollständig entleert werden oder nicht, ist wohl demnach nicht als erledigt zu betrachten.

A priori ist es nicht wahrscheinlich, dass die Ventrikeln im Stande sind, sich ihres Inhalts vollständig zu entleeren; es geht dies aus folgender Betrachtung hervor. - Zum leichteren Verständniss derselben dient Taf. III, Fig. 5, welche einen Querschnitt eines Ochsenherzens an der Grenze der Atrien und Ventrikeln (in verkleinertem Massstabe) darstellt; a ist das Ostium atrioventriculare sinistrum; b Ostium aortae; c die durchschnittene Valvula bicuspidalis; d Ostium atrioventriculare dextrum; e Conus arteriosus; f Muskelmasse; g Fettlage. - Dass die Basis in der Region des Sulcus transversus während der Systole nicht zusammengezogen wird, ist allgemein angenommen, und die anatomischen Verbältnisse sprechen für eine solche Annahme. Die geräumigen Ostia 
atrioventricularia sind bekanntlich von fibrocartilaginösen Ringen umgeben, und die Muskelsubstanz ist in der Region der Basis, wie aus der Figur ersichtlich, rings herum von einer sehr dicken Fettlage (g) bedeckt. Wenn man dabei bedenkt, dass die Atrien während der Systole gefüllt und die Ostia arteriosa von Blut sogar ausgedehnt sind, so darf man wohl mit voller Sicherheit diese Partie als eine im Wesentlichen unveränderliche betrachten. Sollten nun die Höhlungen der Herzkammern während der Systole vollständig verschwinden, so miisste dieses, wenn die Basis unbeweglich ist, entweder dadurch geschehen, dass das Herz unmittelbar unter dem Sulcus transversus durch die Contraction der Muskelsubstanz rund herum so stark zusammengeschnürt würde, dass die inneren Flächen der Aussenwände der Ventrikeln theils gegen die Ostia hinaufgebracht, so dass diese vollständig bedeckt wären, theils in Contact mit dem Septum gebracht wïrden, oder auch dadurch, dass die Herzspitze sich so stark nach oben gegen die Basis zöge, dass die Wände der Herzkammern alle Ostia verschlössen.

Im ersten Falle wiurde das Herz am Ende der Systole ungefähr wie Fig. 7 auf Tafel III aussehen. Diese Figur stellt einen combinirten Quer- und Frontalschnitt eines Ochsenherzens in verkleinertem Massstabe dar; fg der transverselle Schnitt durch die Basis von vorn nach hinten bis zur Mitte derselben; $\mathbf{b} \mathrm{g}$ der Frontalschnitt von der Spitze gegen die Basis, mit dem Vorhergehenden zusammenstossend; i $\mathbf{k}$ zeigen die zusammengeschnürten Regionen; $\mathrm{m}, \mathrm{n}, \mathrm{o}$ und $\mathrm{p}$ die inwendigen Flächen der äusseren Wände, welche also theils ( $m, n)$ die Ostia decken, theils (o p) in Contact mit dem Septum S sich befinden. Das Zusammensehnüren ist durch Bänder erzeugt worden, welche um das Herz ungefähr einen Zoll unterhalb der Basis straff gekntipft wurden.

Im zweiten Falle würde das Herz nicht bloss von dem Apex zu der Basis stark verkürzt, sowie von einer Seite zur anderen stark erweitert, sondern mit der Spitze bedeutend gegen die eine Seite verschoben werden ${ }^{1}$ ).

1) Nach diesen Hauptarten kann man sich verschiedene Modificationen vorstellen, z. B. dass das Einschnüren nicht gleichmässig auf dem ganzen Umkreise geschehe, sondern dass vorzugsweise die Wände an zwei oder mehreren Stellen hineingetrieben würden. Man mag sich aber diese Modificationen vor- 
Wenn man aber ein lebendiges Herz während der Contraction betrachtet, so kann man sich leicht davon überzeugen, dass solche Formveränderungen nie vorkommen. Die Muskelsubstanz zieht sich deshalb auf keine dieser Weisen zusammen, und die Kammern können daher aller Wahrscheinlichkeit nach ihren Inhalt nicht vollständig entleeren.

Um nun genauere Aufschlüsse zu erhalten, wurde die Raumverkleinerung während der Todtenstarre untersucht. Bei stark ausgesprochenem Rigor mortis ist die ganze Muskulatur des Herzens so stark zusammengezogen, dass die Cavitäten aller Wahrscheinlichkeit nach hier in bedeutenderem Grade abnehmen, als bei der gewöhnlichen Systole. Man müsste deshalb vermuthen, dass die Kammern in diesem Zustande geschlossen sein könnten, wenn diese überhaupt im Stande sein sollten, ihren ganzen Inhalt zu entleeren. Unsere Erfahrungen haben indess ergeben, dass ein vollständiges Verschliessen ni cht stattfindet.

Bei Versuchen, welche zu diesem Zwecke angestellt worden sind, wurden frisch herausgenommene Ochsenherzen so lange aufbewahrt, bis die Todtenstarre ihr Maximum erreicht hatte; durch die Atrien wurde dann ohne Anwendung von Druck flüssige Injectionsmasse eingefiuhrt, und die Herzen wurden in eine starke Kältemischung (ca. $-17^{\circ} \mathrm{C}$.) gelegt, damit die Form beibehalten werden konnte, und die Injectionsmasse steif wurde. Gewöhnlich 12 Stunden nachher wurden sie herausgenommen und waren steif gefroren, so dass man leicht mittelst eines starken, diünblättrigen, scharfen Messers eine Reihe von Querschnitten machen konnte. Das constante Resultat sämmtlicher Versuche war nun, dass selbst bei dem stärksten Rigor mortis sowohl in der linken wie in der rechten Kammer Raum zurückblieb.

Um eine klare Darstellung davon zu geben, auf welche Weise die Raumverkleinerung vor sich geht, dienen die Abbildungen eines todtenstarren, injicirten und steifgefrorenen Herzens mit Querschnitten in acht verschiedenen Höhenlagen der Kammern (Taf. IV and Taf. V). A (Taf. V) stellt ein Ochsenherz in starkem Rigor mortis in $2 / 3$ der natürlichen Grösse dar; a, b, c, d, e, f, g, h (Taf. IV und Taf. V) repräsentiren die acht Querschnitte. Die Quer-

stellen, wie man will, in jedem Falle kommen Formen zum Vorschein, welehe der Wirklichkeit nicht entsprechen. 
schnitte a, b, c gehen nur durch die linke Kammer, welche bei a vollkommen geschlossen ist, bei b in der Mitte eine runde Oeffnung von der Grösse eines Stecknadelkopfes zeigt, bei c eine Oeffnung, die mehr oval and wenigstens doppelt so gross ist. In den Querschnitten d, e, f, g, h, wo $\mathrm{v}$ der offenstehende Raum der linken Kammer ist, sieht man denselben stätig an Grösse gegen oben hin zunehmen, so dass die ganze Höhle annähernd einen Conus (freilich sehr unregelmässig) mit der Spitze nach unten bildet. (Vergl. Taf. V, Fig. 2, welche genau die Form der in der linken Kammerhöhle erstarten Injectionsmasse in $2 / 3$ ihrer Grösse wiedergiebt.) Die rechte Kammer wird, wie man sieht, auch nicht geschlossen. Aus den Querschnitten $d, e, f, g, b$ ersieht man, dass der offenstehende Raum (k), welcher anch hier einigermassen gleichmässig gegen den oberen Theil zunimmt, ein halbmondförmiges Aussehen hat. Aus diesem Grunde wird hier die Cavität schmäler, nimmt abei andererseits eine grössere Ausdehnung an als die der linken Kammer ${ }^{1}$ ). Fig. 3, 'Taf. $V$ giebt genau die Form der in der rechten Kammerhöhle erstarrten Masse in $2 / 3$ ihrer Grösse wieder.

In dem Vorhergehenden dürfte demnach zur Genüge dargethan sein, dass die Cavitäten nicht geschlossen werden können, indem selbst unter einer starken Todtenstarre nicht unbedeutende Räume in ihnen zur ückbleiben. Hiervon kann man sich auch unmittelbar überzeugen, wenn man während des Rigor mortis einen oder zwei Finger in die Höhlen durch die Ostia atrioventricularia hineinsteckt.

Es muss also am Ende der Systole ein nicht ganz geringes Quantum Blut in den Kammern zurïckbleiben.

Eine andere Frage ist es, ob man aus den mitgetheilten Versuchen berechtigt ist, einen bestimmten Schluss betreffs des Grades der Raumverkleinerung während der Systole zu ziehen. In dieser Beziehung kann zur Zeit nur gesagt werden, dass die Wahrseheinlichkeit dafür spricht, dass die zurïckbleibenden Räume hier eher grösser als kleiner sein müissen.

Die Querschnitte geben namentlich in dem unteren Theile

1) Man sieht, dass die Höhlen sowohl der linken wie der rechten Kammer in ihrem oberen Theile freistehende Sehnenfäden (ch. t. in den Querschnitten $e, f$ and $g$ ) enthalten. 
eine klare Vorstellung, auf welche Weise die Muskelsubstanzen in den beiden Kammern sich zusammenlegen; man sieht in $a$, wo das Schliessen complet ist, verzweigte Falten, welche in den übrigen Querschnitten wie Strahlen von den Ecken der unverschlossenen Räume ausgehen. Die Verkleinerung der Cavitäten der Kammern während der Contraction geschieht also sowohl da, wo sich die Wände vollständig zusammenlegen, als auch an den Stellen, wo ein Raum übrig bleibt, durch ein Zusammenfalten der Innenwände.

B) Die Papillarmuskeln.

Mehrere Physiologen haben angenommen, dass die Papillarmuskeln beim Schliessen der Atrioventrieularklappen eine ganz besondere Rolle spielen. Man hat nämlich die Behauptung aufgestellt, dass diese Muskeln den Klappenschluss begünstigen sollten, indem man sich wesentlich daran hielt, dass eine und dieselbe Papillarmuskel die angrenzenden Theile zweier Läppchen mit Sehnenfäden versieht. Dass die Papillarmuskeln an und für sich eine solehe Wirkung hervorbringen könnten, ist schon deshalb wenig wahrscheinlich, weil sie die Klappen nicht in ihrem ganzen Umfange mit Sehnenfäden versehen; eine nicht geringe Anzahl letzterer namentlich in der rechten Herzkammer (das linke Läppchen der Valvula tricuspidalis) entspringt nämlich direct aus der Herzsubstanz selbst.

Andere Physiologen haben sogar behaupten wollen, dass die Papillarmuskeln während ihrer Contraction nicht bloss das Schliessen begïnstigen, sondern auch gleichzeitig die Klappen so weit nach unten ziehen sollten, dass die Atrien gewissermassen auf Kosten der Ventrikeln erweitert würden. Aber in diesem Falle wiürden offenbar die Klappen in drei verschiedenen Richtungen, nämlich einwärts, aufwärts und abwärts bewegt werden, und dies dürfte wenig wahrscheinlich sein.

Um nun aber in diesen Fragen zu grösserer Klarheit zu gelangen, ist zunächst eine genaue Berücksichtigung der anatomischen Verhältnisse nothwendig.

Die Hauptpartien der Papillarmuskeln stehen mit der eigentlichen Herzmuskulatur in inniger Verbindung, indem sie integrirende Theile ${ }^{1}$ ) derselben ausmachen; es sind dagegen nur

1) In dem Menschenherzen zeigen sich die hier besprochenen integrirenden Theile der Papillarmuskeln freier, jedoch gleichwohl so innig durch 
die kleineren Partien der Papillarmuskeln, welche frei hervorspringen; vergl. Taf.III, Fig. 8 und Taf. V, Fig. 4, welche zwei der Länge nach durchschnittene Papillarmuskeln im rechten (Taf. V, Fig. 4) and im linken (Taf. III, Fig. 8) Ventrikel des Ochsenherzens in verkleinertem Massstabe darstellen; $a$ ist die frei hervorspringende kleinere Partie, $b$ die Hauptpartie.

Aus dieser Verschiedenheit in anatomischer Beziehung entstehen auch verschiedene Wirkungen.

Die Hauptpartien müssen wegen ihrer innigen Verbindung mit der Herzmuskulatur allen Bewegungen derselben folgen, und die Beantwortung der Frage, inwiefern die Valvulae von diesen Partien nach oben oder nach unten geführt werden können, hängt demnach vollkommen davon ${ }_{z} \mathrm{ab}$, ob die letzteren durch die Contraction der Ventrikeln den Ostia atrioventricularia genähert oder von denselben entfernt werden. Im zweiten Falle wïrden sie nämlich ein Abwärtsziehen der Klappen bewirken können, in ersterem Falle dagegen nicht. Von diesen beiden Möglichkeiten spricht eine iiberwiegende Wahrscheinlichkeit (wenn nicht Gewissheit) für den ersten Fall, also dafuir, dass die Klappen jedenfalls nicht von diesen Partien abwärts gezogen werden können.

Anders stellt sich das Verhältniss betreffs der frei vorspringenden Partien; ihre Contraction muss an und für sich einen selbständigen Einfluss ausüben und nothwendigerweise bewirken, dass sich dieselben von den Ostia atrioventricularia entfernen. Hiernach könnte man vielleicht annehmen, dass die Klappen von diesen Partien nach unten gezogen wirden, aber eine solche Annahme würde zu rasch sein.

Die Wirkung ihrer Contraction darf nämlich nicht isolirt, sondern nur in Verbindung mit derjenigen der Hauptpartien betrachtet werden. Da nun die letzteren sich selbst und somit auch die freien Partien nach oben gegen die Ostia atrioventricularia bringen, wird man leicht einsehen, dass es die Resultirende dieser beiden entgegengesetzten Bewegungen ist, welche entscheidet, ob die Klappen von den Papillarmuskeln (in ihrer Totalität) nach oben gezogen werden sollen oder nicht.

Um sich nun Kenntniss von dieser Resultirenden verschaffen zu

nach allen Richtungen gehende Trabekeln mit der Herzmuskulatur verbunden, dass die Eintheilung in zwei Partien auch hier ihre Berechtigung hat. 
können, wurden todtenstarre Ochsenherzen untersucht; während eines stark ansgesprochenen Rigor mortis muss man nämlich voraussetzen, dass das Herz einigermassen proportional in allen seinen Muskelpartien zusammengezogen wird und demnach eine Basis für die bezïglichen Schlussfolgerungen abgibt.

Aus den angestellten Untersuchungen (mehr als 30) ergab sich als constantes Resultat, dass die Klappen hier stark nach unten gezogen ${ }^{1}$ ) und vollständig offen waren. Die Ostia atrioventricularia standen sogar so offen, dass man ungehindert zwei Finger durch dieselben bis binunter zu den Papillarmuskeln stecken konnte. Die Resultirende der Contraction der beiden Partien der Papillarmuskeln, ging also während der Todtenstarre in der Richtung nach unten.

Hieraus lässt sich jedenfalls folgern, dass die Contraction der Papillarmuskeln ein Schliessen der Klappen nicht zur Folge haben kann. Andererseits darf man aber aus den hier gewonnenen Resultaten keineswegs ohne Weiteres schliessen, dass die Papillarmuskeln zum Oeffnen der Klappen dienen sollten, also dass ihre Function die gerade entgegengesetzte sei.

Bei der Bestimmung der Function derselben muss man nämlich vor Allem die Verhältnisse während der Systole zu Grunde legen. Hier sind nach allen exacten Beobachtungen (Chauveau, Faivre u. a.) die Klappen immer nach oben getrieben, mit anderen Worten, die Resultirende geht in entgegengesetzter Richtung von der bei der Todtenstarre.

Diese Nichtübereinstimmung findet jedoch leicht ihre Erklärung, wenn man bericksichtigt, dass während der Systole ein Moment vorhanden ist, welches bei dem $\mathrm{Rig}$ or mortis ${ }^{2}$ ) nicht vorkommt, nämlich der Druck des Blutes. Wenn nun die Resultirenden während dieser beiden Zustände des Herzens in

1) Das Abwärtsziehen kann, wie es scheint, vielleicht theilweise von der Contraction des Gewebes sowohl in den Klappen wie in den Chordae tendineae herrïhren. Nach den Observationen zu urtheilen, spricht die Wahrscheinlichkeit dafür, dass auch in diesen Theilen Phänomene auftreten, welche mit dem Rigor mortis analog sind. Dieses verdient eine nähere Untersuchung.

2) Von kaum nennenswerther Bedeutung ist es wohl, dass die Contraction während des Rigor mortis mehr einer ausgeprägt tetanischen als einer systolischen entspricht. 
entgegengesetzter Richtung gehen, so beweist dies nur, dass der Druck des Blutes gregen die Valveln in der Richtung nach oben so stark ist, dass ihm gegenuiber das Abwärtsziehen der Papillarmuskeln ganz and gar in den Hintergrund tritt. Hiernach verdienen die zwei Behauptungen, nämlich:

1) dass die Papillarmuskeln (selbstständig) zum Schliessen der Klappen beitragen

und

2) dass sie ausserdem dieselben nach unten ziehen sollten, wohl kaum eine eingehendere Widerlegung.

Die Function der Papillarmuskeln muss deshalb eine andere sein.

Wenn man die überaus sorgfältige Verschlussweise der Atrioventricularklappen betrachtet und dabei berücksichtigt, wie sowohl die Klappen als auch die Sehnenfäden mit exquisit elastischen Eigenschaften ausgeriistet sind, mit anderen Worten, wie es also hier der Natur darum zu thun war, für einen sicheren und mit elastischen Eigenschaften ver'sehenen Schliessungsmechanismus Sorge zu tragen, so scheint auch die Annahme nahe zu liegen, dass die oben besprochenen frei en Partien der Papillarmuskeln den übrigen Theilen des Verschlussmechanismus beigegeben sind, um die elastischen Eigenschaften desselben zu veryollkommen und vermittelst der Muskelcontractilität eine Regulirung einzuführen.

Dass derartige Eigenschaften von Wichtigkeit sind, geht aus folgenden Betrachtungen hervor. Zum leichteren Verständniss derselben dient Taf. III, Fig. 9, welche einen Frontalschnitt durch den rechten Ventrikel und das rechte Atrium eines Ochsenherzens (bei kïnstlich verschlossener Klappe) in verkleinertem Massstabe darstellt.

r s eine Linie durch das Ostium atrioventriculare.

K K Annulus fibrocartilagineus.

v v Valvula.

11 die umgebogenen Ränder der Klappenzipfel.

$\mathrm{m} n, \mathrm{x} y$ die (freilich willkührlich gezogenen) Grenzen, innerhalb welcher ein gentigend dichter Verschluss voraussichtlich stattfindet.

C C Chordae tendinae.

P P die freien Partien der Papillarmuskeln. 
430 C. Sandborg u. Worm Müller: Studien üb. d. Mechanismus d. Herzens.

B B die Basis dieser freien Partien.

S Septum ventriculare.

$\mathrm{Da}$ die Herzcontractionen mit ungleicher Stärke vor sich gehen, und da Annulus fibrocartilagineus (K K), an welchem die peripherischen Partien der Valvula haften, als unbeweglich angesehen werden kann, so werden die Grundflächen (B B) der freien Partien der Papillarmuskeln während der Systole bald näher bei, bald entfernter von dem Ostium zu liegen kommen, aber hieraus resultirt auch, dass, wenn die freien Partien (P P) der Papillarmuskeln, die Chordae tendineae (C C) and die Valvula ( $\mathrm{V} \mathrm{v}$ ) steif oder unelastisch wären, also dieselben Längen behielten, auch die umgebogenen Ränder (1 l) der Klappenzipfel bald über bald unter der Linie $\mathrm{r}$ s sich befinden würden.

Kämen nun die umgebogenen Ränder (1 l) der Klappenzipfel ausserhalb der Grenzen $\mathrm{mn}, \mathrm{x}$ y zu liegen, oder wenn jedes 1 auf seine Seite von der Linie $\mathrm{r} s$ käme, welches letztere während unregelmässiger Herzcontractionen denkbar ist, so würde leicht ein unvollkommenes Schliessen stattfinden.

Die Nothwendigkeit einer einigermassen genauen Einstellung von den umgebogenen Rändern 11 in die Linie $\mathbf{r}$ s ist also einleuchtend. Damit dies auf eine zweckmässige Weise geschehen kann, sind sowohl Elasticität wie Contractilität (also die Muskelelemente) der Verschlusstheile nothwendig: Die elastischen Eigenschaften werden nämlich kleinere Druckdifferenzen ausgleichen und den Klappenschluss vervollkommnen, während die Muskelelemente durch ihre Fähigkeit, sich je nach den Umständen stärker oder schwächer zasammenzuziehen, speciell zur rechten Einstellung von den umgebogenen Rändern 11 dienen werden. Die Function der Papillarmuskeln besteht also in einer Accommodation, welche von ihren freien Partien ausgeführt wird. 

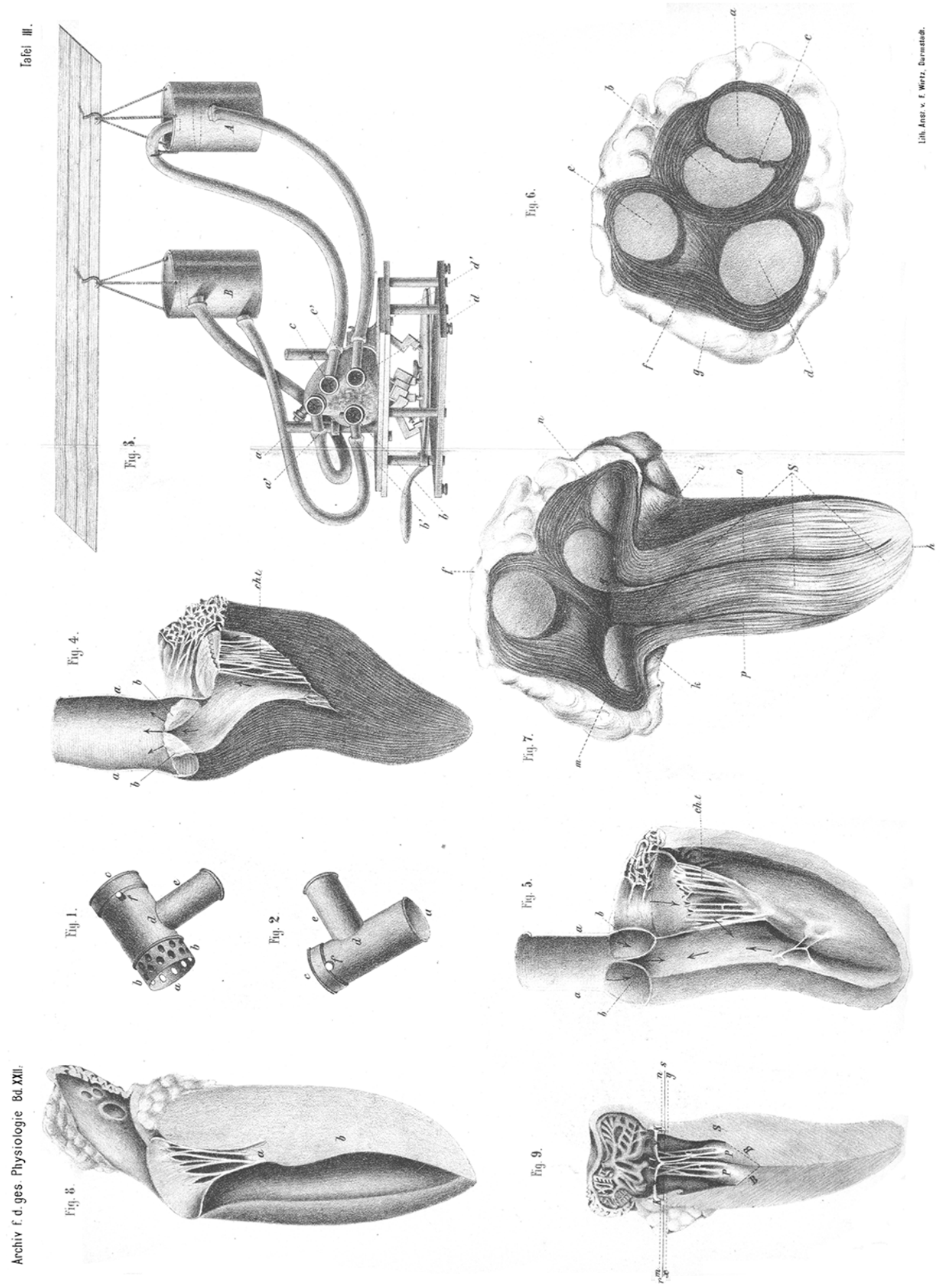

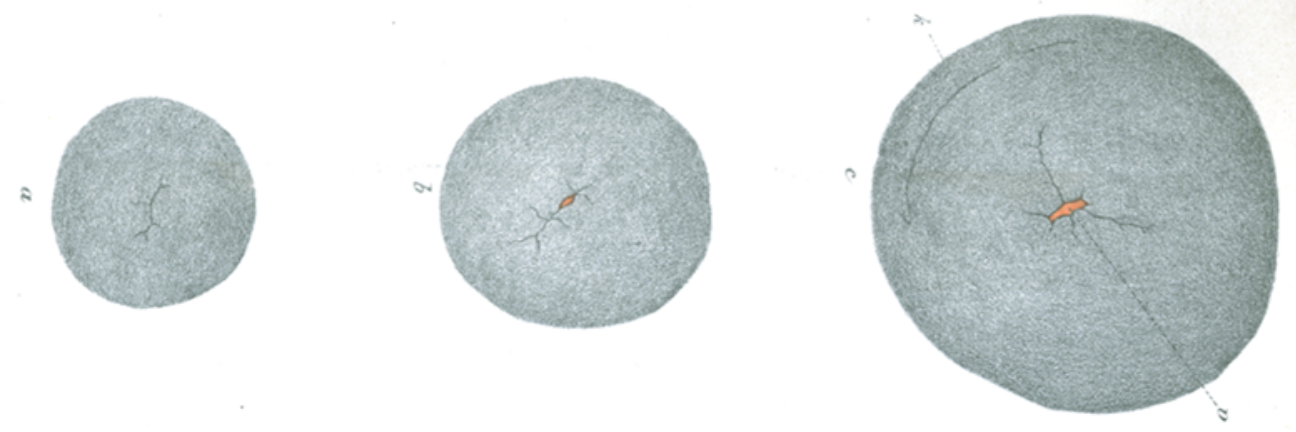

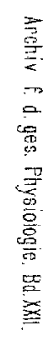
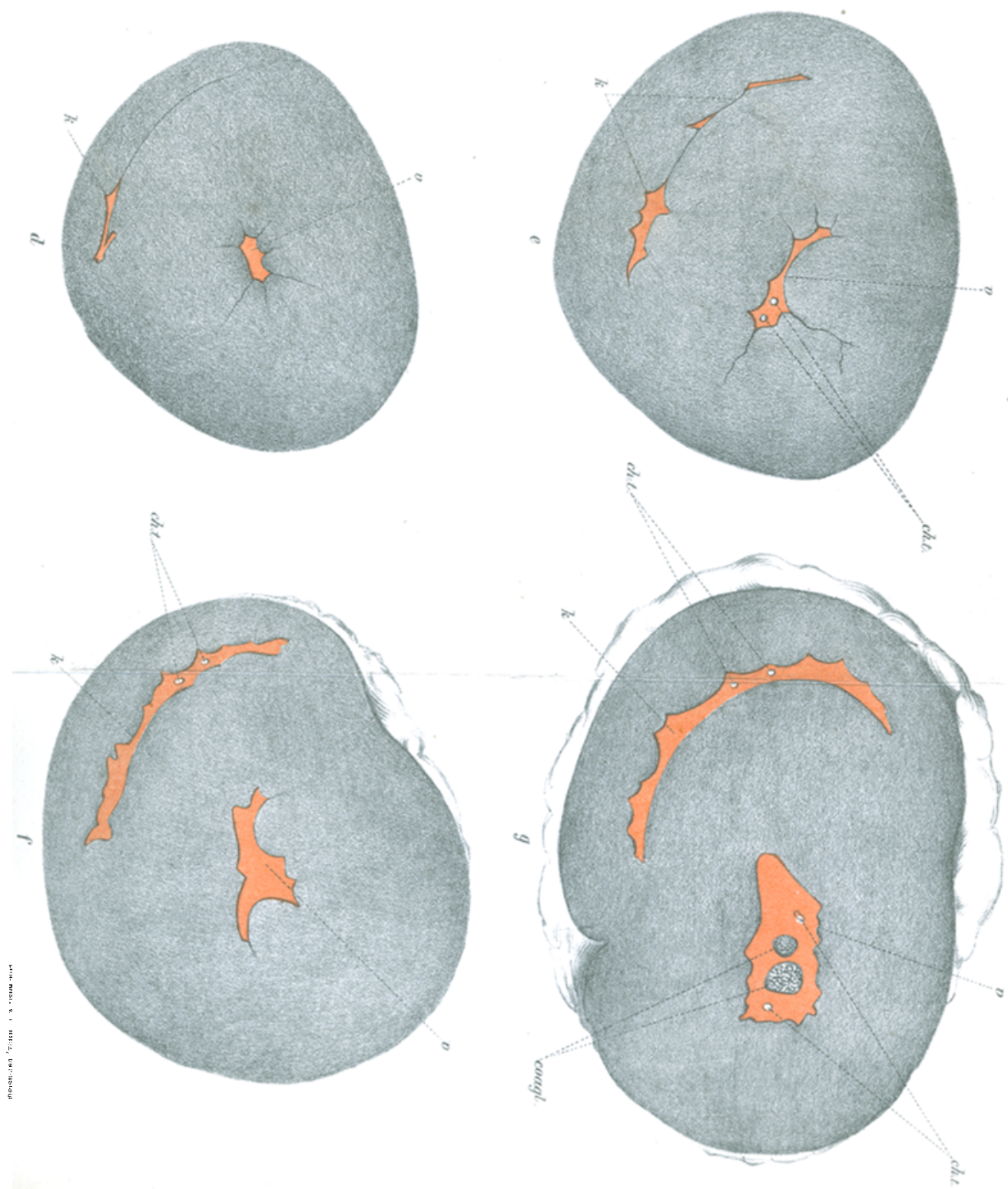

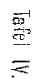



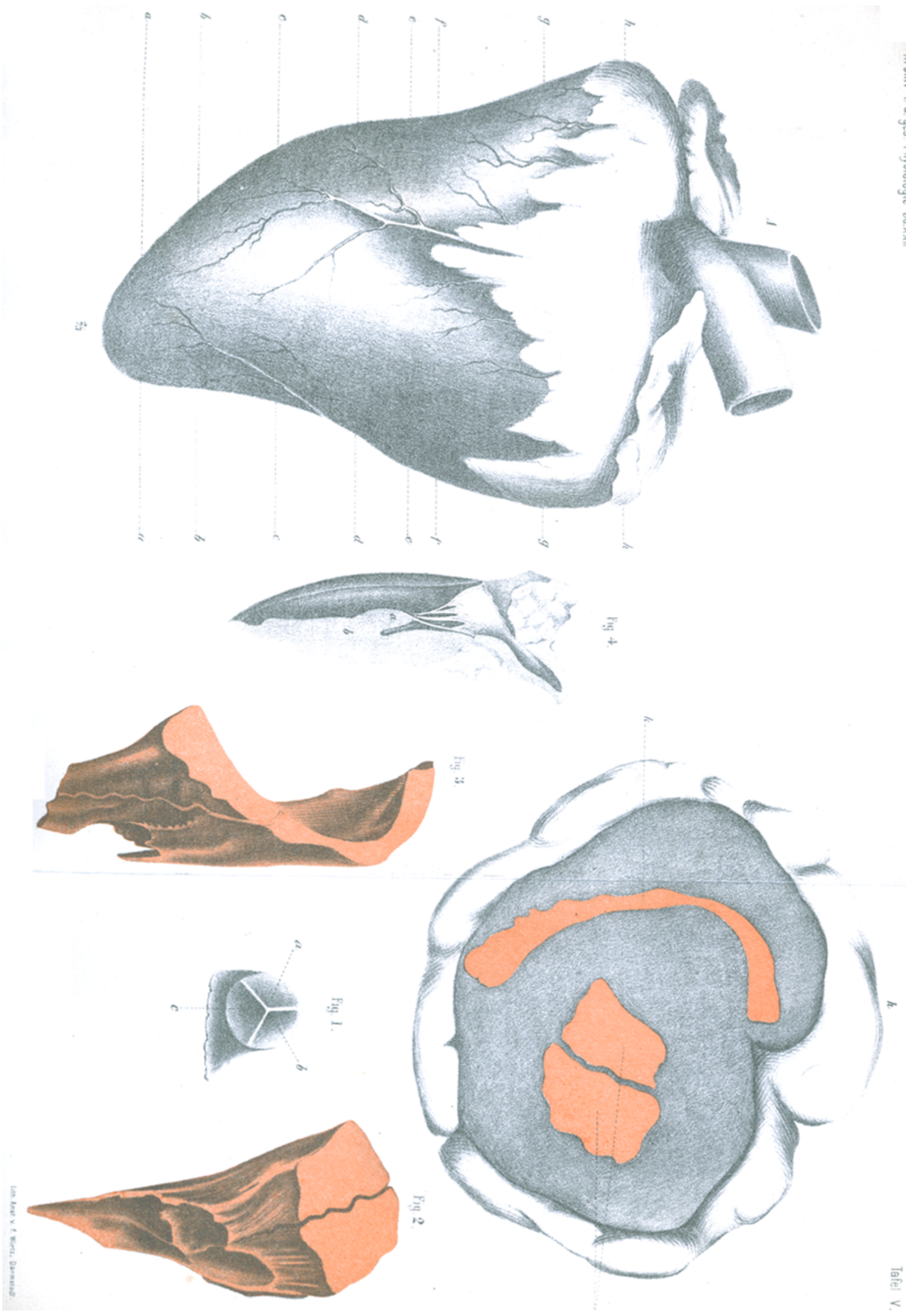